\title{
Absolute sensitivity to white noise under auxiliary visual stimulation'
}

\author{
GARY G. BOTHE ${ }^{2}$ AND LAWRENCE E. MARKS ${ }^{3}$ \\ JOHN B. PIERCE FOUNDA TION LABORATORY AND YALE UNIVERS.'TY SCHOOL OF MEDICINE
}

\begin{abstract}
Absolute sensitivity to white noise was measured with the $O$ in the dark, in constant illumination, and in the dark but with sound-synchronized flashes of light at three different intensities. $A$ confidence rating procedure was used, and the results were analyzed in terms of the theory of signal detectability. There appeared to be no consistent effect of auxiliary visual stimulation on absolute auditory sensitivity for the four Os examined.
\end{abstract}

Studies of the effects of auxiliary visual stimulation on absolute auditory sensitivity have not totally agreed on their results. Most studies have shown that auxiliary white light improves auditory sensitivity (Child \& Wendt, 1938; Gregg \& Brogden, 1952; London, 1954; Özbaydar, 1961; Thompson, Voss, \& Brogden, 1958). On the other hand, other investigators have found that concomitant stimulation with certain colored lights can depress auditory sensitivity (see London, 1954). Sheridan, Cimbalo, Sills, and Alluisi (1966) reported that white light had no effect on auditory sensitivity at four frequencies examined $(250-2000 \mathrm{~Hz})$, but depressed sensitivity at a fifth frequency $(6000 \mathrm{~Hz})$. In none of the abovementioned studies, however, was there evidence of any attempt to control or measure false positive responses. Thus, one possible source of variability is criterion, which might differ with and without auxiliary visual stimulation (see Treisman, 1964).

The present study aimed to evaluate the effects of white light on the detectability of white noise. The conditions compared were darkness, constant room illumination, and sound-synchronized flashes of light at three intensities. Use of a confidence rating procedure and analysis in terms of the theory of signal detectability provided an opportunity to separate any effect of auxiliary visual stimulation on auditory

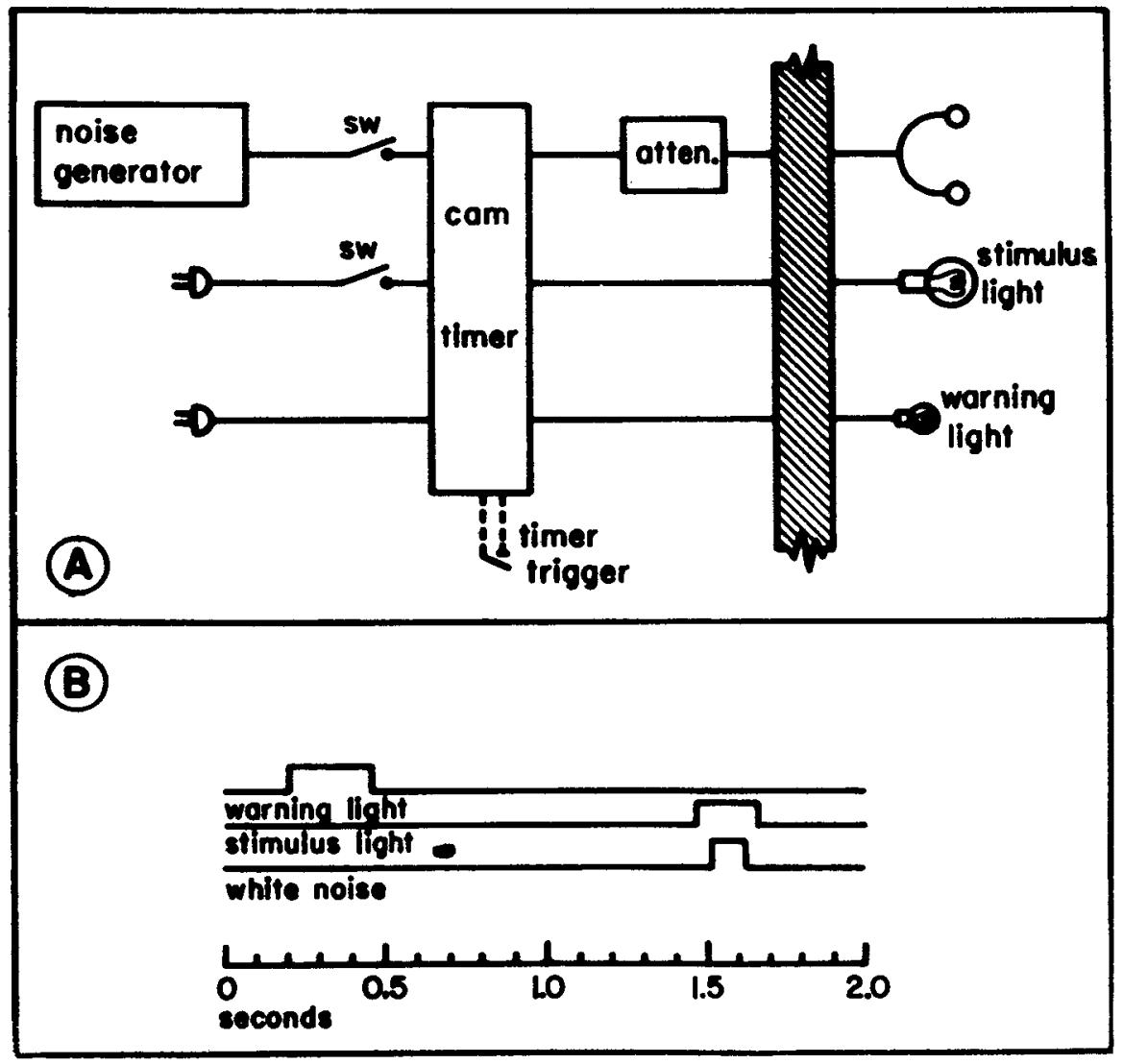

sensitivity from an effect on criterion. This could be accomplished by comparing the entire receiver-operating-characteristic (ROC) for all of the conditions (Green \& Swets, 1966).

\section{Apparatus}

METHOD

Figure la is a schematic diagram of the equipment. The $O$ listened with earphones (Grason-Stadler D30) in a sound-deadened room (IAC). The output of a noise generator (Grason-Stadler 455C) was led through an SPST switch, multiple cam timer, and decade attenuator to the earphones.

Flashing light was provided by a $100-\mathrm{W}$ incandescent lamp mounted in a light-tight container with a $3.8 \mathrm{~cm}$ diam opal glass aperture located at eye level and $1 \mathrm{~m}$ in front of the seated $O$ (visual angle of target, $2.2 \mathrm{deg}$ ). Luminance was controlled by means of neutral filters placed in front of the aperture. Current to the lamp passed through an SPST switch and the cam timer. A small, dim warning light, also controlled by the cam timer, was placed $10 \mathrm{~cm}$ above the flashing light. Constant room illumination could be produced by two $100-\mathrm{W}$ lamps mounted on a wall of the test chamber.

Triggering the timer illuminated the warning light for $250 \mathrm{msec}$ (see Fig. 1b). One second after the offset of the warning light current to the stimulus light was turned on for $200 \mathrm{msec}$. The white noise was turned on $50 \mathrm{msec}$ after the onset of the light and remained on for $100 \mathrm{msec}$.

\section{Procedure}

The $O$ was seated in the darkened experimental room with earphones in place. In a preliminary session, the detectability of white noise was determined in the dark by the method of constant stimuli. The results of this preliminary session were used to select a stimulus level to be maintained for all conditions of the experiment.

The study employed five conditions of auxiliary visual stimulation: warning light followed by 1800,18 , and $1.8 \mathrm{c} / \mathrm{m}^{2}$ flash, warning light only in darkened test chamber, and warning light only in lighted

Fig. 1 (a) Diagram of the apparatus. (b) Temporal arrangement of the stimuli. 


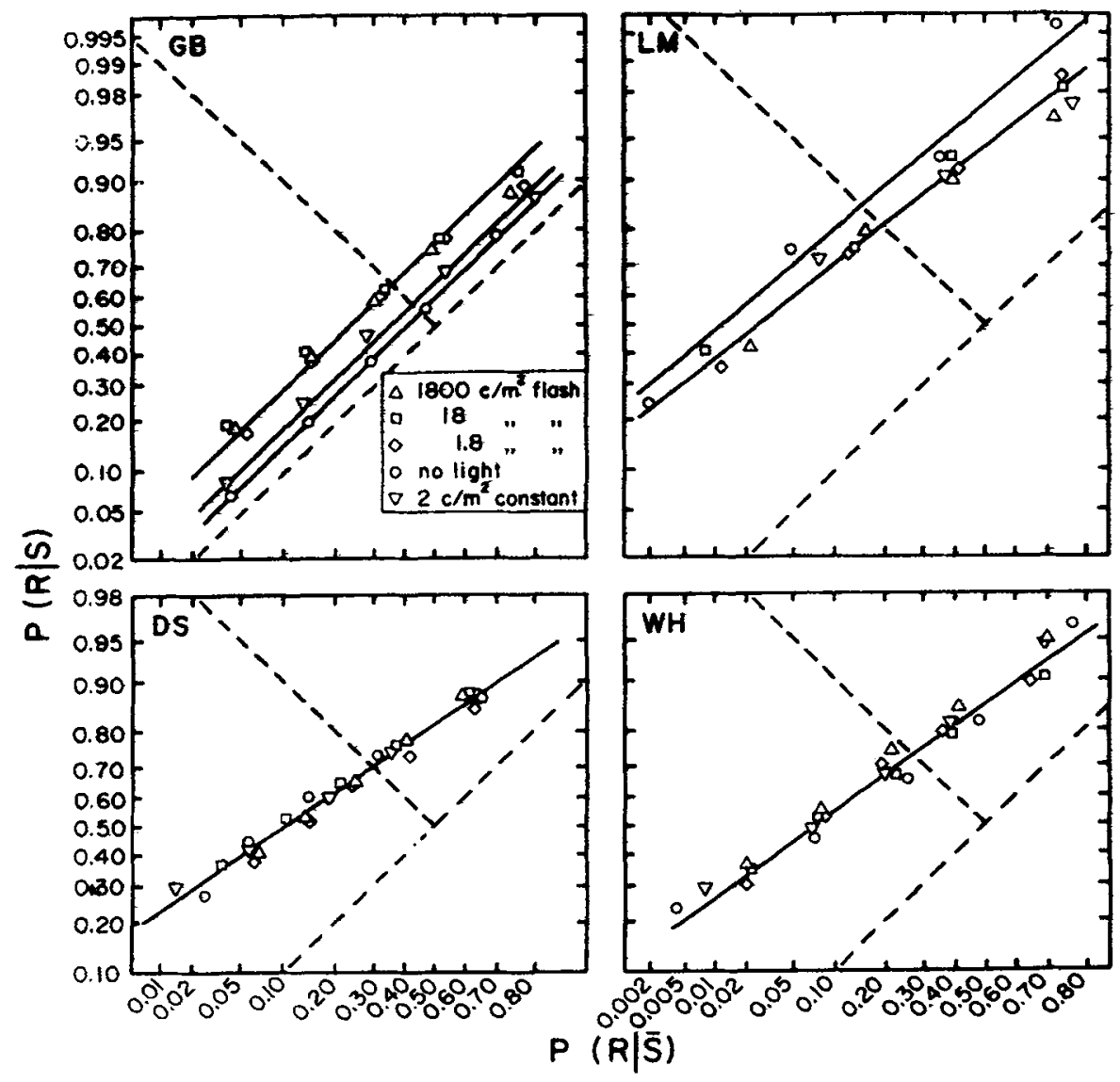

test chamber (about $2 \mathrm{c} / \mathrm{m}^{2}$ in O's field of view).

Each session consisted of a single experimental condition. To acquaint $O$ with the condition, 20 "preview" trials were presented, of which the first and third groups of five trials did contain the white noise signal and the second and fourth groups did not. The $O$ was told that the trials following would contain signal (S) and no-signal $(\bar{S})$ presentations randomly ordered. After each trial $\mathbf{O}$ indicated his degree of certainty that the signal was present by giving one of the integers from 1 to 6,1 indicating certainty that the signal was present and 6 indicating certainty that the signal was not present. Intermediate numbers indicated in termediate degrees of certainty.

The $O$ was then given 10 practice trials for which responses were not counted. Following this, 100 test trials were given, equally and randomly divided between $S$ and $\bar{S}$ presentations. Each experimental session lasted $15 \mathrm{~min}$, and $O$ rested for $15 \mathrm{~min}$ between sessions. All five experimental conditions were presented on a single day in irregular order with the exception that neither the no-light nor the constant-light condition was presented first to two of the Os (DS and WH). The five conditions were run on ten different days. Thus, for each condition each $O$ was given
$500 \mathrm{~S}$ and $500 \overline{\mathrm{S}}$ presentations. Four men, including the authors, served as Os.

\section{RESULTS}

The ROC curves are plotted in the double-probability coordinates of Fig. 2. The experimental points were calculated by the method described by Green and Swets (1966); this method treats each boundary between response categories as a separate criterion level. The results for the four Os are more strikingly different than similar. GB showed greatest sensitivity in the three flashing-light conditions $\left(d^{\prime}=0.72\right)$, lower sensitivity with constant light $\left(d^{\prime}=0.39\right)$, and lowest sensitivity with no light $\left(d^{\prime}=0.23\right)$. $L M$, on the other hand, showed greatest sensitivity with no light $\left(d^{\prime}=2.08\right)$ and lower sensitivity in the four light conditions $\left(d_{e}^{\prime}=1.74\right){ }^{4} \mathrm{DS}$ and WH showed practically no differences at all in sensitivity among experimental conditions $\left(\mathrm{d}_{\mathrm{e}}^{\prime}=1.05\right.$ and 1.23 , rerespectively).

The slope of the ROC curves also varies from one $O$ to another. GB gave slopes approximately equal to unity; a unit slope is consistent with the assumption that the underlying distributions are Gaussian and of equal variance. DS and WH, however, gave slopes smaller than unity (0.71 and 0.73 , respectively); such slopes are consistent with the assumption that the
Fig. 2 ROC curves for four Os.

underlying S-distribution has a greater variance than the underlying $\overline{\mathbf{S}}$-distribution. LM also gave slopes smaller than unity ( 0.85 for the no-light condition and 0.80 for the four light conditions).

\section{DISCUSSION}

Although most studies have found that auxiliary visual stimulation enhances absolute auditory sensitivity, at least a few studies have reported decreased sensitivity or no effect. The present results appear to demonstrate all three of the above: GB showed lowest sensitivity when no light was present; LM showed lowest sensitivity when light was present; DS and WH showed essentially no differences among the five conditions.

Egan, Schulman, and Greenberg (1961) have shown that when temporal uncertainty is large, sensitivity is poor. Thus, an auxiliary stimulus such as flashing light can improve sensitivity by serving as a temporal indicator. In the present experiment, however, such an effect was probably diminished by regular use of a warning light. Even so, GB's results show enhancement under flashing light; it appears that the flashing light served as a temporal indicator. ${ }^{5}$

In conclusion, it would appear that the effect of simultaneous visual stimulation on absolute auditory sensitivity is idiosyncratic. To the extent that effects may occur, we suspect their basis to be non-"sensory." Decreased sensitivity may well be due to distraction or disturbance; increased sensitivity may be due primarily to reduction of temporal uncertainty.

\section{REFERENCES}

CHILD, I, \& WENDT, G. R. The temporal course of the influence of visual stimulation upon the auditory threshold. Joumal of Experimental Psychology, 1938, 23, 109-127.

EGAN, J. P., SCHULMAN, A. I., \& GREENBERG, G. Z. Memory for waveform and time uncertainty in auditory detection. Journal of the Acoustical Society of America, $1961,33,779-781$.

GREEN, D. M., \& SWETS, J. A. Signal detection theory and psychophysics New York: Wiley, 1966.

GREGG, L. W., \& BROGDEN, w. J. The effect of simultaneous visual stimulation on absolute auditory sensitivity. Journal of Experimental Psychology, 1952, 43, 179-186.

LONDON, I. D. Research on sensory interaction in the Soviet Union. Psychological Bulletin, $1954,51,531-568$.

OZBAYDAR, $S$. The effects of darkness and light on auditory sensitivity. British Journal of Psychology, 1961, 52, 285-291.

SHERIDAN, J. A., CIMBALO, R. S., SILLS, J. A., \& ALLUISI, E. A. Effects of darkness, constant illumination, and synchronized photic stimulation on auditory sensitivity to pulsed tones. Psychonomic Science, 1966, 5, 311-312. 
THOMPSON, R. F., VOSS, J. F., \& BROGDEN, W. J. Effect of brightness of simultaneous visual stimulation on absolute auditory sensitivity. Journal of Experimental Psychology, 1958, 55, 45-50.

TREISMAN, M. The effect of one stimulus on the threshold for another: An application of signal detectability theory. British Journal of Statistical Psychology, 1964, 17, 15-35.

\section{NOTES}

1. This research was supported by Contract F44620-67-C-0017 with the Air Force Office of Scientific Research.
2. Present address: Department of Psychology, Florida State University, Tallahassee, Florida 32306.

3. Address: John B. Pierce Foundation Laboratory, 290 Congress Avenue, New Haven, Connecticut 06519

4. The measure $d_{e}$ is defined as twice the value of the normal deviate at the intersection of the ROC curve and the negative diagonal. Whep the slope of the ROC curve equals unity, $d_{e}^{\prime}=d$.

5. A similar observation was made in some of DS's preliminary sessions. When DS's first run was the no-light or constant-light condition, performance was relatively poor. No differences occurred among conditions when the first run was a flashing-light condition. Thus, a little practice with flashing light early in the day appeared to continue its effect later. It was for this reason that neither the no-light nor the constant-light condition was presented first on any day to DS or to WH.

(Accepted for publication November 17, 1969.) 